Article

\title{
Anomalous Behaviors of Spin Waves Studied by Inelastic Light Scattering
}

\author{
Hua-Yi Hou ${ }^{1}$, Ming Yang ${ }^{1}$, Jin Qiu ${ }^{2}$, In-Sang Yang ${ }^{2, *}$ and Xiang-Bai Chen ${ }^{1, * \mathbb{C}}$ \\ 1 School of Science and Hubei Key Laboratory of Optical Information and Pattern Recognition, \\ Wuhan Institute of Technology, Wuhan 430205, China \\ 2 Department of Physics, Ewha Womans University, Seoul 03760, Korea \\ * Correspondence: yang@ewha.ac.kr (I.-S.Y.); xchen@wit.edu.cn (X.-B.C.)
}

Received: 30 May 2019; Accepted: 10 July 2019; Published: 14 July 2019

\begin{abstract}
Magnonics, an emerging research field, aims to control and manipulate spin waves in magnetic materials and structures. However, the current understanding of spin waves remains quite limited. This review attempts to provide an overview of the anomalous behaviors of spin waves in various types of magnetic materials observed thus far by inelastic light scattering experiments. The anomalously large asymmetry of anti-Stokes to Stokes intensity ratio, broad linewidth, strong resonance effect, unique polarization selection, and abnormal impurity dependence of spin waves are discussed. In addition, the mechanisms of these anomalous behaviors of spin waves are proposed.
\end{abstract}

Keywords: spin wave; anti-Stokes; linewidth; resonance; polarization; magnonic crystal

\section{Introduction}

Magnonics, a young and evolving field, has received extensive research interests in the modern magnetism research community [1-20]. Similar to spintronics, which deals with electron spin, magnonics utilizes magnon (the quanta of spin wave) to carry and process information. The field of modern magnonics is very wide and covers a variety of emerging physical phenomena. One of the fundamental issues would be understating the properties of propagating spin waves, which are considered as promising ideal information carriers, due to their following advantages. The propagation of spin waves is free of charge transport, and hence can avoid Joule heating induced energy loss in nowadays micro- or nano- electronic devices; also spin waves are less subject to dissipation caused by scattering with impurities on the atomic level because of the charge current free property. In addition, spin waves can be easily manipulated by an applied magnetic field, and in magnetoelectric multiferroics which possess coexisting magnetic and ferroelectric phases, they can be manipulated by applied an electric field [21-29]. Moreover, for same frequency spin wave and electromagnetic wave, the wavelength of spin wave is several orders of magnitude shorter. Spin wave can be in the terahertz frequency with sub-nanometer wavelength, thus very promising for miniaturization. Furthermore, magnonic devices can be easily integrated with electronic and photonic devices. For the development of modern magnonic devices, it would be necessary to have a thorough understanding of spin waves and how they behave in various materials and structures.

Spin waves are normally investigated through four experimental methods: spin wave resonance (ferromagnetic resonance) [30-34], inelastic electron scattering (spin-resolved electron energy loss spectroscopy) [35-39], inelastic neutron scattering [40-46], and inelastic light scattering (Brillouin scattering and Raman scattering) [47-60]. Among these methods, inelastic light scattering would be the most convenient approach for studying spin waves, especially Raman scattering spectroscopy. Raman spectroscopy, a powerful, fast, and non-destructive experimental technique, has been extensively applied not only in basic scientific researches, but also for practical applications. Usually, Raman 
spectroscopy is applied for studying vibrational properties of materials. However, due to the anomalous behaviors of spin wave scattering comparing with vibrational scattering, Raman spectroscopy study of spin wave is difficult and limited. In this paper, we present a review of anomalous behaviors of spin waves observed by inelastic light scattering experiments, especially by Raman spectroscopy. The large asymmetry of anti-Stokes to Stokes intensity ratio, broad linewidth, strong resonance effect, unique polarization selection, and abnormal impurity dependence of spin waves are discussed, and a proposed model for the mechanisms of spin flip, spin relaxing, and spin wave scattering is presented for understanding these anomalous behaviors of spin waves. In addition, two magnonic crystal structures are proposed for manipulating spin waves through analyses of the abnormal impurity dependence of spin waves. The review of the anomalous behaviors of spin waves and the proposed models provide the directions for easier experimental study of spin waves by inelastic light scattering, which will be useful for raising the research efforts for investigating spin waves. This is important for the emerging research of magnonics, which has received extensive interests in the modern magnetism research community.

\section{Anti-Stokes to Stokes Intensity Ratio}

Inelastic light scattering interaction with material can lead to two possible outcomes: (i) the material absorbs energy and the emitted photon has a lower energy than the absorbed photon, this outcome is labeled as Stokes inelastic light scattering; and (ii) the material loses energy and the emitted photon has a higher energy than the absorbed photon, this outcome is labeled as anti-Stokes inelastic light scattering. For light scattering of crystal vibrations, the intensity ratio of anti-Stokes to Stokes scattering can be simply modeled by considering the thermal population factor $\exp \left(-\hbar \omega / k_{\mathrm{B}} T\right)$, i.e.,

$$
\frac{\mathrm{I}_{\mathrm{AS}}}{\mathrm{I}_{\mathrm{S}}}=\exp \left(-\frac{\hbar \omega}{k_{\mathrm{B}} T}\right)
$$

where $\omega$ is the frequency of the phonon mode, $k_{\mathrm{B}}$ is the Boltzmann's constant, and $T$ is the temperature. However, for spin wave scattering, it had been found that a modification of the anti-Stokes to Stokes intensity ratio from the usual thermal factor $\exp \left(-\hbar \omega / k_{\mathrm{B}} T\right)$ should be considered [51,52], i.e.,

$$
\frac{\mathrm{I}_{\mathrm{AS}}}{\mathrm{I}_{\mathrm{S}}}=\operatorname{Aexp}\left(-\frac{h \omega}{k_{\mathrm{B}} T}\right)
$$

where the parameter A would be bigger than 1 . The value of A depends on the sample property, and external experimental conditions, such as, applied magnetic field, laser wavelength, laser polarization, scattering geometry, etc. [61-68].

The large asymmetry of anti-Stokes to Stokes intensity ratio of spin wave scattering was first predicted by Cottam [51] in studying the interaction of light with antiferromagnet. It was discussed that the inclusion of a magneto-optical coupling quadratic in the sublattice magnetisation operators should significantly change the anti-Stokes to Stokes intensity ratio of spin wave scattering from the usual thermal factor $\exp \left(-\hbar \omega / k_{\mathrm{B}} T\right)$. The quadratic magneto-optical coupling model had been mostly adopted for interpreting the anomalous anti-Stokes to Stokes intensity ratio of spin wave scattering. However, the quadratic magneto-optical coupling model cannot explain the phenomenon of anti-Stokes to Stokes intensity ratio of spin wave scattering in various magnetic materials, such as, $\mathrm{FeBO}_{3}, \mathrm{LuFeO}_{3}, \mathrm{Fe}, \mathrm{Ni}$, Permalloy, etc. $[63,69,70]$. In addition, the quadratic magneto-optical coupling model could not explain the asymmetry behavior when external magnetic field is applied [52,63]. Camley et al [63] proposed that the large asymmetry is due to the contribution of the off-diagonal spin-spin correlation function to the light scattering intensity. However, this contribution would be only seen when the wave vector of the light in the ferromagnet is complex, the case for absorptive materials.

Another possible contribution of the asymmetry of anti-Stokes to Stokes intensity ratio of spin wave scattering could be correlated with the presence of surface spin wave mode. Experimental 
studies showed that surface spin wave has two anomalous asymmetry properties [71-75]. First, surface spin wave comes either Stokes or anti-Stokes depending on the way the sample is being tilted or the direction of applied magnetic field. Second, the Stokes and anti-Stokes of surface spin wave is reversed when the sample is rotated $180^{\circ}$ around the optical axis or when the applied magnetic field is reversed. Thus, if surface spin wave is observed together with bulk spin wave, and their frequency is similar. Then, the two anomalous asymmetry properties of surface spin wave would have significant contribution on the asymmetry behavior of bulk spin wave. However, it had been shown that surface spin wave generally has frequency quite different as that of bulk spin wave (surface spin wave has frequency higher than that of bulk; unlike phonon and plasmon, a lower frequency is found), and the condition of observing surface spin wave is limited [71-75]. In addition, it was reported that the large asymmetry of anti-Stokes to Stokes intensity ratio in $\mathrm{Fe}, \mathrm{Ni}$, Permalloy could not be explained by the localization of surface spin wave [63]. Therefore, the general property of asymmetry of anti-Stokes to Stokes intensity ratio of bulk spin wave scattering should not be correlated with the anomalous asymmetry properties of surface spin wave.

In recent years, magnetic excitations in magnetoelectric multiferroics have been attracted much research interests [76-89]. The magnetic excitations in multiferroic hexagonal manganites have been systematically investigated through resonance and polarization Raman scattering experiments $[81,82,87,89]$. The resonance Raman scattering study indicated that the spin wave scattering in hexagonal manganites can be selectively excited with red lasers, which is correlated with the resonance condition of on-site Mn d-d transition (more details of resonance selection is discussed in Section 4) [82]. The polarization selection study indicated that the spin wave scattering in hexagonal manganites can be selectively excited under $\mathrm{z}(\mathrm{yx}) \underline{\mathrm{z}}$ polarization configuration, but not detectable under $\mathrm{x}(\mathrm{yz}) \underline{\mathrm{x}}$ and $\mathrm{y}(\mathrm{zx}) \mathrm{y}$ polarization configurations, this is also the polarization condition of on-site Mn d-d transition (more details of polarization selection is discussed in Section 5) [89]. The resonance and polarization studies of spin wave in hexagonal manganites indicated that the on-site $\mathrm{Mn} \mathrm{d}-\mathrm{d}$ transition would be the first step to excite the spin wave scattering, thus a physical picture of the processes of spin flip, spin relaxing, and spin wave scattering in hexagonal manganite was proposed [89]. The generalized model of the processes of spin flip, spin relaxing, and spin wave scattering in magnetic materials can be schematically presented in Figure 1. A microscopic picture of the model in Figure 1 would be as follows. (i) In the electronic ground state of a magnetic ion, spin flip will be accompanied with the excitation of magnetic ion from electronic ground state to electronic excited state; while without the electronic excitation of the magnetic ion, spin flip is difficult; i.e., spin wave scattering would be difficult between the spin ordered state and spin flipped state of electronic ground state of the magnetic ion. (ii) During the spin relaxing, the spin flipped magnetic ion interacts with the neighboring magnetic ions, which changes only the direction of neighboring spin vectors, and thus forming spin wave propagating in the magnetic material. (iii) In inelastic light scattering experiments, the observed spin wave would be originated from scattering between the spin ordered state and spin flipped state of electronic excited state of the magnetic ion.

Our proposed model in Figure 1 provides a good explanation of the anomalous asymmetry characters of anti-Stokes to Stokes intensity ratio of spin wave scattering. Firstly, Figure 1 proposed that spin wave is originated from scattering of electronic excited states of the magnetic ion. Thus, the anti-Stokes to Stokes intensity ratio would generally differ considerably from the usual thermal factor $\exp \left(-\hbar \omega / k_{\mathrm{B}} T\right)$. Secondly, Figure 1 suggests that the asymmetry of anti-Stokes to Stokes intensity ratio would depend on the spin relaxing process, thus the asymmetry would be different in different magnetic materials. For fast spin relaxing, small asymmetry is expected; and for slow spin relaxing, large asymmetry will be observed. Thirdly, the electronic transition and spin relaxing would be affected by external conditions, such as, laser excitation, polarization, applied electric field, applied magnetic field, etc. Thus the asymmetry of anti-Stokes to Stokes intensity ratio would also depend on these external conditions. To verify our proposed model in Figure 1, extensive experimental and theoretical studies would be needed. 


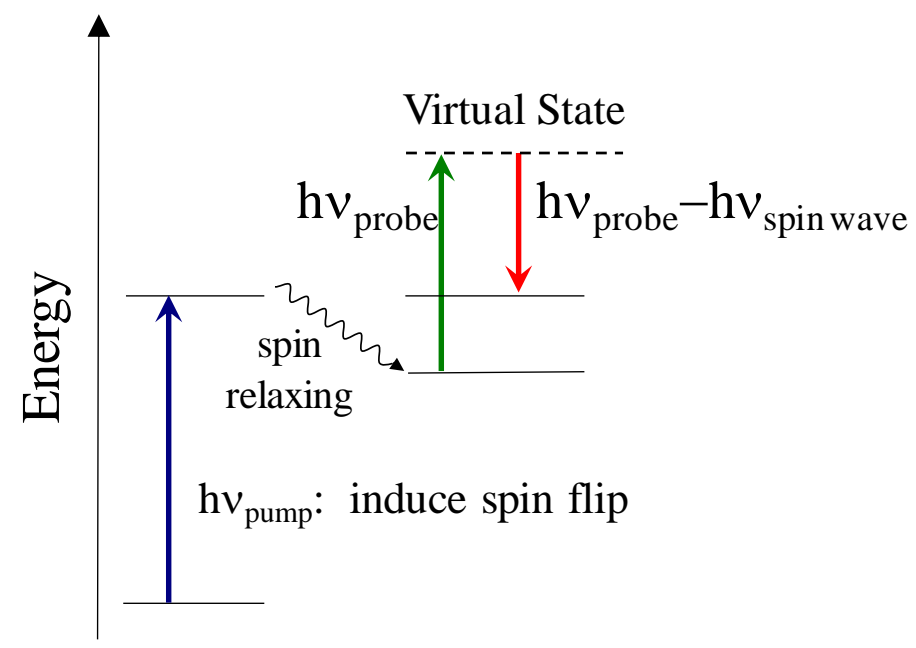

Figure 1. Schematic energy diagram of the processes of spin flip, spin relaxing, and spin wave scattering. In the figure, Stokes spin wave scattering is presented.

Experimentally, time resolved study would be very helpful to approve the model in Figure 1 and investigate the properties of spin waves. Figure 1 suggested that in magnetic crystals, Stokes spin wave scattering would have two more steps of spin flip and spin relaxing comparing with Stokes phonon scattering. Thus Stokes spin wave emission would occur much slower than Stokes phonon emission, which occurs nearly simultaneously with the excitation of laser light. A schematic temporal profile of the laser pulse, Stokes phonon scattering and Stokes spin wave scattering is presented in Figure 2. The model in Figure 2 indicates that in experimental measurement, spin wave emission time should be carefully differentiated with the lifetime of spin wave. The spin wave emission time is the response time of spin wave emission after laser excitation, the lifetime of spin wave is correlated with the intrinsic linewidth of spin wave scattering. These two times could be in same order, different as that of phonon scattering. For phonon scattering, the emission time would be a few orders shorter than the lifetime. More discussion of the difference of spin wave life time and response time will be presented in Section 3 with respect to broad linewidth, which is correlated with short lifetime of spin wave. In time resolved experimental study, appropriate laser pulse and temporal gate would be able to differentiate the difference of phonon and spin wave emission, thus approve the model in Figure 1. Also, the time resolved study would be helpful to understand the dynamics of spin wave scattering process. In addition, the model in Figure 1 indicated that for Stokes scattering of spin wave, the response time would be significantly longer than lifetime; while for anti-Stokes scattering of spin wave, the response time would be only somewhat longer than lifetime. This suggests that a systematic time resolved study of the Stokes and anti-Stokes of phonon and spin wave scattering would be helpful to approve the model in Figure 1 and investigate the properties of spin waves.

In addition to the time resolved study, pump-probe study would also be very helpful to approve the model in Figure 1 and investigate the properties of spin waves. For hexagonal manganites, Figure 1 predicted that when a sample is pumped in the resonant condition (i.e., excitation source with energy $\sim 1.7 \mathrm{eV}$ ), the spin wave (i.e., magnon peak at $\sim 800 \mathrm{~cm}^{-1}$ ) could still be detected with a probe excitation even in the non-resonant regime. Furthermore, Figure 1 indicated that when ultrafast pulsed excitation sources are applied, the time dependence of anti-Stokes to Stokes intensity ratio of spin wave scattering would be very useful for studying the dynamics of spin flip, spin wave, and their relaxations in magnetic materials. 


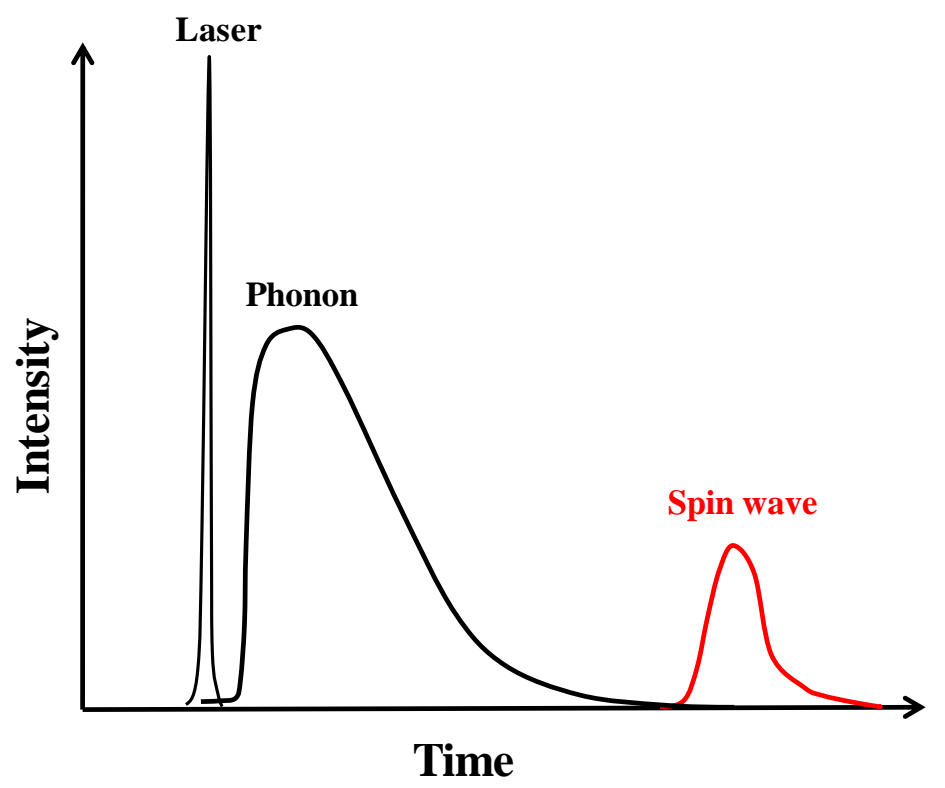

Figure 2. Schematic temporal profile of laser excitation, phonon scattering, and spin wave scattering. In the figure, the difference of Stokes phonon and Stokes spin wave is presented.

The proposed model in Figure 1 not only explains well the anomalous asymmetry of anti-Stokes to Stokes intensity ratio of spin wave, but also explains well the anomalous properties of broad linewidth, strong resonance effect, and unique polarization selection of spin waves. These anomalous properties are discussed in the following sections.

\section{Broad Linewidth}

In general, the theory of a spectral line shape of a signal in a dispersive medium predicts the linewidth, a parameter describing the damping effect, to be inversely proportional to the lifetime of the signal [90]. For inelastic light scattering of crystal vibration, the intrinsic linewith is correlated with the phonon lifetime. One fundamenal lifetime shortening mechamism of phonon has been established to occur via anharmonic interaction [91,92]. Moreover, the impurities and defects had been found affecting phonon lifetime [93]. The linewidth of spin wave is not well understood comparing with that of crystal vibration. Experimentally, it had been found that the linewidth of spin wave can differ significantly in magnetic materials. Broad linewith of spin wave had been observed in many magnetic mateirals, such as, $5 \mathrm{~d}$ pyrochlore $\mathrm{Cd}_{2} \mathrm{Os}_{2} \mathrm{O}_{7}$, spin-orbit Mott insulators $\mathrm{Sr}_{2} \mathrm{IrO}_{4}$ and $\mathrm{Sr}_{3} \mathrm{Ir}_{2} \mathrm{O}_{7}$, copper-oxide based high temperature superconductors, hexagonal manganites, etc. [58,59,89,94-96]. However, it is difficult to understand the mechanism of broad linewidth of spin wave scattering $[89,95]$.

The conventional theory of spin wave scattering had been performed by Elliott and Thorpe [97], and Parkinson [98]. However, it predicted much narrower spin wave linewidth (at least a factor of 3) than experimental results in cuprate-oxide based high temperature superconductors. A few models had been given for explaining the broad linewidth of spin wave, including spinon model, phenomenological damping, quantum fluctuation, cyclic exchange contributions, etc. [94,99-101]. However, these models could only explain the broad linewidth of spin wave in certain types of magnetic materials. Our recent Raman spectroscopy studies of spin wave in hexagonal manganite thin films and single crystals indicated that the broadness of spin wave has very complex origin [89].

The broad linewidth behavior of spin wave scattering could be well understood by the model in Figure 1. First, the lifetime of spin wave would be correlated with the spin relaxing process. For fast spin relaxing, spin wave would have short lifetime thus broad linewidth; and for slower spin relaxing, spin wave would have longer lifetime thus narrower linewidth. In different types of magnetic materials, the spin relaxing process could differ significantly, thus the linewidth differs significanlty. 
Second, Figure 1 indicated that spin wave would be originated from scattering of excited electronic states of magnetic ion. In excited state, generally spin relaxing process would be fast, thus spin wave would have short lifetime, i.e., broad linewidth. These are consistent with the experimental studies of spin wave lifetime, which had shown that the lifetime is generally much shorter than picoseconds (the phonon lifetime is generally in the order of picoseconds) $[96,102,103]$. In metallic ferromagnet, spin wave lifetime longer than picosencod was reported [104,105]. As discussed in Section 2, this anomalous long lifetime could be actually the response time of spin wave emission, which can be in the picoseconds region.

Figure 1 also indicated that the broadness of spin wave linewidth would have a correlation with the asymmetry of the anti-Stokes to Stokes intensity ratio. For narrower linewidth, i.e., slower spin relaxing, more significant asymmetry of the anti-Stokes to Stokes intensity ratio would be expected, since the population of spin flipped electrons would differ more significantly from the usual thermal factor $\exp \left(-\hbar \omega / k_{\mathrm{B}} T\right)$. Thus, a systematic understanding of the model in Figure 1 and study of the correlation between spin wave linewidth and anti-Stokes to Stokes asymmetry would be helpful for further investigation of broad linewidth mechanism of spin wave scattering.

In addition to the anomalously broad linewidth of spin wave in a single magnetic material, our recent studies showed anomalous behaviors of spin wave linewidth and lineshape between $\mathrm{LuMnO}_{3}$ single crystal and $\mathrm{HoMnO}_{3}$ thin film $[87,89]$. The spin wave linewith is much broader and the lineshape is much more asymmetric in single crystal comparing with that in thin film. A direct comparison of spin wave scattering between hexagonal $\mathrm{LuMnO}_{3}$ single crystal and $\mathrm{HoMnO}_{3}$ thin film is presented in Figure 3. The $\mathrm{A}_{1}$ phonon mode of hexagonal $\mathrm{LuMnO}_{3}$ single crystal did show narrower linewidth than that of hexagonal $\mathrm{HoMnO}_{3}$ thin film. This narrower linewidth would due to better crystalline quality and smaller instrumental bandpass broadening. However, the spin wave of hexagonal $\mathrm{LuMnO}_{3}$ single crystal showed broader linewidth than that of $\mathrm{HoMnO}_{3}$ thin film. In addition, the spin wave of hexagonal $\mathrm{LuMnO}_{3}$ single crystal is much more asymmetric than that of $\mathrm{HoMnO}_{3}$ thin film, shoulder peaks on both higher and lower energy sides were observed. These indicate very complex origin of spin wave linewidth and lineshape.

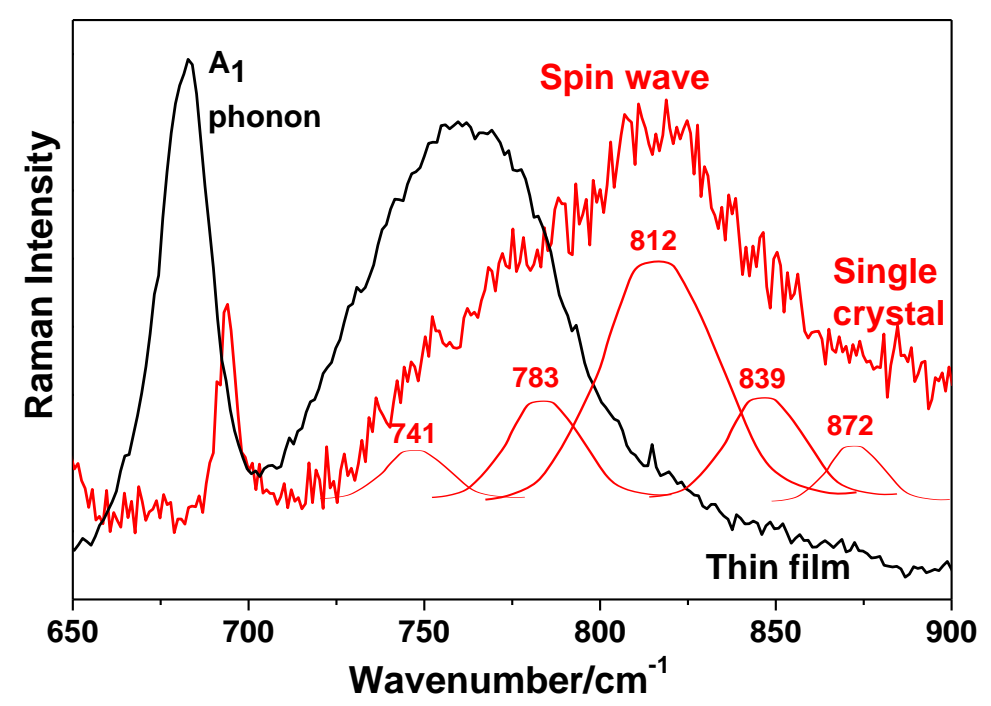

Figure 3. Comparison of spin wave scattering in hexagonal $\mathrm{LuMnO}_{3}$ single crystal and $\mathrm{HoMnO}_{3}$ thin film. The spectrum of $\mathrm{LuMnO}_{3}$ single crystal is from Figure 1 of Ref. [89], the spectrum of $\mathrm{HoMnO}_{3}$ thin film is from Figure 1 of Ref. [81].

The complex origin of the differences of spin wave linewidth and lineshape between hexagonal $\mathrm{LuMnO}_{3}$ single crystal and $\mathrm{HoMnO}_{3}$ thin film could be correlated with the differences of crystal quality, rare earth element, resonance condition, laser probe depth, etc. A 2-Dimentional Correlation Spectroscopy study of hexagonal $\mathrm{LuMnO}_{3}$ single crystal showed that the broad spin wave peak is 
constituted of five individual peaks at $741,783,812,839$, and $872 \mathrm{~cm}^{-1}$ [88]. Also, the asymmetric lineshape of the broad spin wave peak in Figure 3 indicated that it would constitute multiple peaks. These multiple peaks could be due to better crystalline quality of single crystal, special property of $\mathrm{Lu}$ rare earth, stronger resonance condition, and longer laser probe depth in the $\mathrm{LuMnO}_{3}$ single crystal sample, thus more possible spin wave modes with similar energy could be excited.

The possibility of multiple spin wave modes with similar energy could also be explained with the model in Figure 1. We propose that in resonant excitation of magnetic ion, the accompanied spin flip would occur in all the electrons which contributed for the magnetic moment of magnetic ion; and in the spin relaxing process, a spin flipped electron could relax individually or together with other spin flipped electrons, thus producing different types of spin wave modes propagating in the magnetic ion network. A clear picture of possible multiple spin wave modes can be understood in the following example of hexagonal manganite. In the electronic ground $\mathrm{d}$ state of $\mathrm{Mn}^{3+}$ ion, four electrons occupy four lowest orbitals giving $m_{s}=2$. The electronic ground $d$ state with $m_{s}=-2$ is difficult to achieve, i.e., spin flip without on-site $\mathrm{d}-\mathrm{d}$ transition is difficult. The spin flip is accompanied with resonant electronic excitation of $d-d$ transition, i.e., the electronic excited $d$ state with $m_{s}=-2$ can be much more easily achieved. This is consistent with the temperature dependent study of on-site d-d transition, which showed that the transition has a blueshift of $\sim 0.1 \mathrm{eV}$ with cooling in the antiferromagnetic state $[106,107]$. This blueshift is attributed to the differences in exchange interaction in the excited and ground states of a given $\mathrm{Mn}^{3+}$ ion [106]. Also the value of blueshift is about the energy of the spin wave at $\sim 815 \mathrm{~cm}^{-1}$, which is correlated with 4-spin-flip mode [89]. Thus, in the resonant excitation of on-site $\mathrm{d}$-d transition, the accompanied spin flip would occur in all the four electrons in $\mathrm{Mn}^{3+}$ ion. During the spin relaxing, the spin flipped electrons in $\mathrm{Mn}^{3+}$ ion could relax individually or together and then interact with the neighboring $\mathrm{Mn}^{3+}$ ions, thus forming different modes of spin waves propagating in hexagonal manganites. For example, possible 4-spin-flip modes could be originated from one $\mathrm{Mn}^{3+}$ ion, or two neighboring $\mathrm{Mn}^{3+}$ ions (each has two spin flip), or four neighboring $\mathrm{Mn}^{3+}$ ions (each has one spin flip), etc. These possible modes would have similar energy, but different scattering cross section. In hexagonal $\mathrm{LuMnO}_{3}$ single crystal, more possible modes could be excited, thus the observed spin wave has anomalously broad linewidth and very asymmetric line shape.

\section{Resonance Effect}

The traditional framework for understanding the resonance effect of spin wave scattering has been known as the Loudon-Fleury theory [108]. From the "Loudon-Fleury" diagrams, spin wave scattering would have resonance enhancement near the band edge [109]. Extensive studies of the resonance effect of spin wave scattering had been performed in cuprate antiferromagnetic materials through Raman spectroscopy [110-113]. However, these studies showed that there is no resonance enhancement of spin wave scattering near the absorption edge, but the resonance effect occurs well above it. To explain this anomalous resonance effect in cuprate insulators, several complex models had been proposed, such as, triple resonance mechanism, excitonic mechanism, background spins mechanism, etc. [111-113].

In our previous Raman spectroscopy study of the resonance effect of spin wave scattering in hexagonal manganite, it was found that the resonance enhancement did occur near the band edge [82], in good agreement with the Loudon-Fleury theory. Hexagonal manganite can be considered as a Mott-Hubbard type material. The theory of Raman scattering in Mott-Hubbard systems has been presented by Shastry and Shraiman [114], which can be derived from the Loudon-Fleury theory. Thus, it would be not surprising that resonance effect of spin wave scattering in hexagonal manganite agrees well with the Loudon-Fleury theory.

The discrepancy of very different resonance mechanism of spin wave scattering between cuprate insulators and hexagonal manganites could be simply correlated with the difference of their electronic structures [82]. For hexagonal manganites, they belong to the Mott-Hubbard type materials, in which the on-site Coulomb energy $U$ (transition between the lower and upper Hubbard bands) is at lower 
energy than the charge-transfer energy $\Delta$. For cuprate insulators, they belong to the charge-transfer type materials, in which the on-site Coulomb energy $U$ is at higher energy than the charge-transfer energy $\Delta$. In hexagonal manganites, the resonance occurs near the band edge, i.e., the on-site Coulomb energy U. In cuprate insulators, it was found that the resonance occurs right at the upper end of the features in the optical data that can be interpreted as the particle-hole excitations between the lower and upper Hubbard bands [111], i.e., also the on-site Coulomb energy U. Therefore, in both cuprate insulators and hexagonal manganites, the resonance enhancement of spin wave occurs near the on-site Coulomb energy.

The strength of resonance enhancement of spin wave scattering is also very different in cuprate insulators and hexagonal manganites. The strength of spin wave resonance in cuprate insulators is much weaker than that in hexagonal manganites. This would be correlated with the linewidth difference of on-site Coulomb energy in cuprate insulators and hexagonal manganites. In hexagonal manganites, on-site Coulomb energy has very narrow linewidth, thus extremely strong resonance enhancement; while in cuprate insulators, the linewidth of on-site Coulomb energy is significantly broader, thus much weaker resonance enhancement. Therefore, the strength of resonance enhancement of spin wave would be correlated the linewidth of on-site Coulomb energy.

Through the comparison of the resonance behaviors of spin wave scattering in cuprate insulators and hexagonal manganites, we proposed a simple general mechanism of resonance enhancement of spin wave. In magnetic materials, the resonance effect of spin wave scattering would occur near the on-site Coulomb energy, and narrower linewidth of the on-site Coulomb energy would have much stronger resonance effect. In a recent study of $\mathrm{V}_{2} \mathrm{O}_{3}$ [115], a canonical Mott-Hubbard insulator at low temperatures, it was also found that the resonance effect of spin wave scattering is correlated with the on-site V 3d-3d Coulomb energy, and the very weak resonance effect is correlated with the very broad linewith of on-site V 3d-3d transition. This further supported our proposed model of the resonance effect of spin wave scattering.

The proposed model in Figure 1 can also explain the resonance enhancement behavior of spin wave scattering. Figure 1 proposed that spin flip should be induced first with resonant excitation of magnetic ion from ground state to excited state, then spin wave scattering is possible. This transition of magnetic ion is correlated with the on-site Coulomb energy. Therefore, Figure 1 indicated that spin wave scattering would be possible when incident photon energy is within the absorption peak of the on-site Coulomb energy, i.e., spin wave has strong resonance enhancement with excitation close to the on-site Coulomb energy, and the strength of resonance would be correlated with the linewidth of the on-site Coulomb energy. This is in good agreement with our above discussion. In addition, the process of spin wave scattering proposed in Figure 1 suggests that spin wave scattering would have stronger resonance enhancement than phonon scattering; and for narrower linewidth of on-site Coulomb energy, spin wave scattering would have more significant resonance enhancement than phonon scattering. This is also in good agreement with our resonant Raman spectroscopy studies of hexagonal manganite and $\mathrm{V}_{2} \mathrm{O}_{3}[82,115]$. In hexagonal manganite, the linewidth of on-site Coulomb energy is very narrow, the resonance enhancement of spin wave scattering is a few orders of magnitude stronger than that of phonon scattering. In $\mathrm{V}_{2} \mathrm{O}_{3}$, the linewidth of on-site Coulomb energy is very broad, the resonance enhancement of spin wave scattering is only a few times stronger than that of phonon scattering.

\section{Polarization Selection}

The polarization selection of inelastic light scattering of crystal vibration had been well studied, which can be understood in term of electronic polarizability tensor [116-121]. A Raman mode of crystal vibration is active if one of the components of the polarizability tensor is changed during the vibration, and it can be observed by inelastic light scattering experiment with choosing appropriate polarizations of incident light and scattered light according to the nonzero component. In a recent Raman spectroscopy study of spin wave scattering in hexagonal manganite single crystal, it was shown that the polarization selection of spin wave is also different comparing with that of crystal 
vibration [89]. For crystal vibration, only electronic polarization needs to be satisfied; while for spin wave, both electronic and magnetic polarizations need to be satisfied, as discussed below.

The Raman spectroscopy study of spin wave in hexagonal $\mathrm{LuMnO}_{3}$ single crystal showed that the spin wave can be excited under $\mathrm{z}(\mathrm{yx}) \underline{\mathrm{z}}$ configuration, but not detectable under $\mathrm{x}(\mathrm{yz}) \underline{\mathrm{x}}$ and $\mathrm{y}(\mathrm{zx}) \mathrm{y}$ configurations, as presented in Figure 4 . This polarization dependence of spin wave scattering from the three planes of hexagonal manganites is different comparing with that of $A_{1}$ and $E_{1}$ phonon modes. Below Néel temperature, the spins of the Mn moments are aligned antiferromagnetically in the basal $a-b$ plane of hexagonal manganites [122-124]. Therefore, Figure 4 suggests that to excite spin wave, both the polarization of electric field and magnetic field should be properly selected; while for phonon scattering, only the polarization of electric field needs to be properly selected. In hexagonal $\mathrm{LuMnO}_{3}$ single crystal, to excite spin wave, the electric field of laser excitation should be perpendicular to the $c$ axis, and the magnetic field of laser excitation should be parallel to the plane of spin alignment. Under $\mathrm{z}(\mathrm{yx}) \underline{\mathrm{z}}$ configuration, $E \perp c$ and $H \| a b$-plane, thus spin wave can be excited. Under $\mathrm{y}(\mathrm{zx}) \mathrm{y}$ configurations, $H \| a b$-plane but $E \| c$, thus spin wave can not be excited. Under $\mathrm{x}(\mathrm{yz}) \underline{\mathrm{x}}$ configuration, $E \bar{\perp} c$ but $H \| z$, thus spin wave can not be excited.

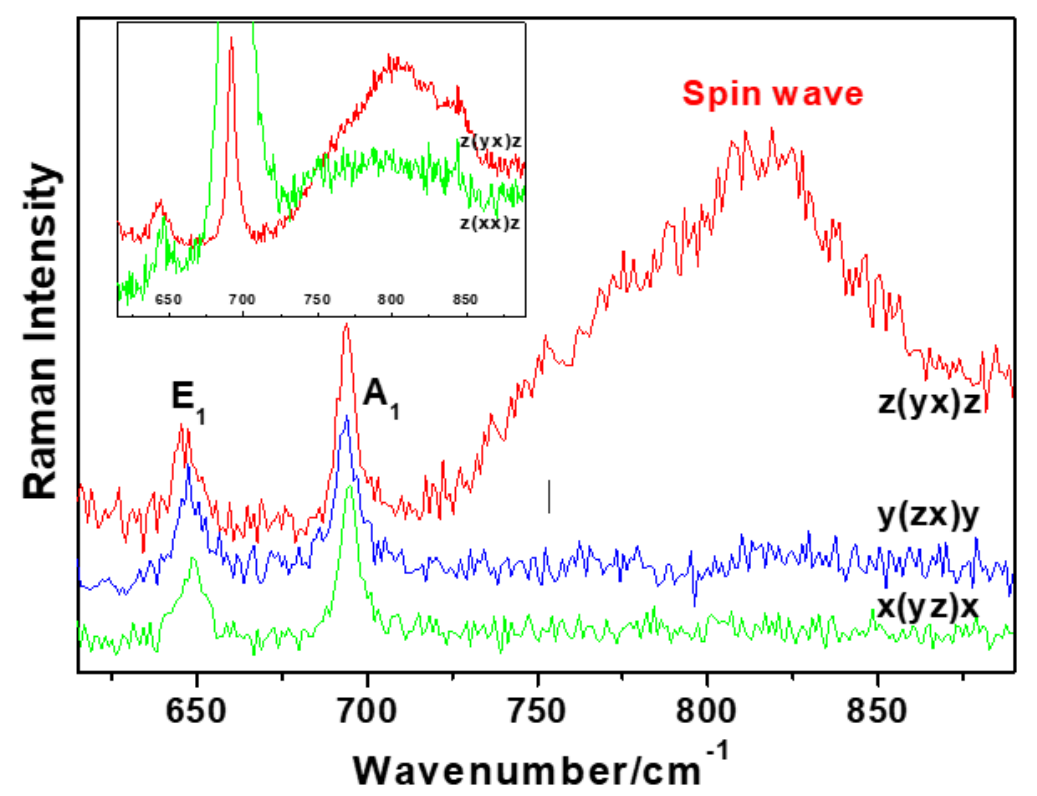

Figure 4. Polarized Raman spectra ofspin wave scattering in hexagonal $\mathrm{LuMnO}_{3}$ single crystal, which is from Figure 1 of Ref. [89]. The inset is from Figure 1 of Ref. [87].

The polarization property of spin wave scattering in the basal a-b plane of hexagonal manganites was investigated in both single crystal and thin film samples [82,89]. It was shown that in back scattering from basal a-b plane, spin wave scattering can be observed in both parallel and cross polarization configurations, as shown in the inset of Figure 4. In addition, the intensity of spin wave scattering is not affected when the excitation laser polarization is rotated around the $c$ axis, and when the collection polarization is changed from parallel to cross configuration (in the inset of Figure 4, the intensity difference of spin wave in parallel and cross configuration is due to the difference of integration time). This would be correlated with the characters of magnetic $\mathrm{Mn}^{3+}$ ions in hexagonal manganite crystal. The on-site Mn $d$ - $d$ transitions are only allowed with $E \perp c$ polarization of light [106]; and the $\mathrm{Mn}^{3+}$ ions forming regular triangular networks in the $a-b$ plane in hexagonal manganite [122-124]. Therefore, the intensity of spin wave scattering in the $a-b$ plane would not change when incident excitation light is rotated.

The polarization dependence of spin wave scattering could also be explained by the model in Figure 1. Figure 1 proposed that spin wave scattering could be observed after the resonant excitation of magnetic ion from ground state to excited state. Thus, the polarization selection must first satisfy 
the polarization condition for exciting the magnetic ion from ground state to excited state. This is in good agreement with the experimental result of polarization dependence of spin wave scattering.

\section{Impurity Dependence}

Manipulate and control of spin waves is a key issue in magnonics. Spin wave is originated from the excitation of spin ordering of magnetic ions. Thus, doping on the magnetic ion sites with other elements could be useful to investigate how the spin wave is affected by impurities, which would be helpful for better understanding of spin wave properties and also for studying how to manipulate and control spin wave. One important aspect of manipulating spin wave is tuning its frequency. The spin wave frequency is directly correlated with the spin exchange interaction in the magnetic crystal, which can be calculated by Heisenberg Hamiltonian: $H=J \sum_{<\mathrm{i}, j}\left(S_{i} \bullet S_{j}\right)$. Here $J$ is the exchange integral which quantitatively characterizes the spin exchange interaction between magnetic ions; $S_{i}$ is the spin on site $i$ and the summation is over the nearest-neighbor magnetic ion pairs. When magnetic ions are replaced by impurity elements, the total spin exchange interactions would be modified in the spin network. Thus, at first sight, doping would be helpful to manipulate the frequency of spin wave. However, it was found that doping is not helpful to tune the frequency of intrinsic spin wave correlated with host magnetic ions, either by magnetic or non-magnetic elements [125-128].

The study of impurity dependence of spin waves had attracted much research attention about half a century ago, both experimentally and theoretically [125-127,129-132]. It was shown that if the host atoms and the impurity atom carry different magnetic moments, there will be local magnetic excitations involving only the impurity atom and the host atoms surrounding it. Thus, for magnetic ion doping, in addition to the intrinsic spin wave, an additional "localized" spin wave is produced localizing at the doping site. For both the intrinsic spin wave and "localized" spin wave, their frequencies are not much affected by the concentration of doping. The frequency of intrinsic spin wave is similar as that without doping, which is determined by the host magnetic ions. The frequency of "localized" spin wave depends on the doping element, which is determined by the spin exchange interaction of the doping magnetic ion with the neighboring host magnetic ions. Although extensively investigated half a century ago, the mechanisms and properties of localization of spin wave still need further systematic studies. In recent years, as the young and evolving field of magnonics receiving more and more research interests in modern magnetism community, the study of localization of spin wave has rekindled much research interests, which has the potential for a new avenue in magnonics [133-137]. In the previous studies of impurity dependence of spin wave, magnonic ion doping and localized spin waves were mainly investigated. Recently, we performed non-magnetic ion doping dependence of spin wave in hexagonal manganite thin films and single crystals. It was found that with non-magnetic ion doping, only intrinsic spin wave is observed since there is no spin exchange interaction of the doped non-magnetic ion with the host magnetic ions, and the frequency of intrinsic spin wave is similar as that without doping [128]. Therefore, doping is not helpful for tuning the frequency of intrinsic spin wave, either by magnetic or non-magnetic ion doping. For magnetic ion doping, although not helpful for tuning spin wave frequency, it is helpful for producing an additional spin wave of different frequency, and the propagation path of the additional spin wave could be controlled by designing the pattern of doped magnetic ions.

Doping could not tune the frequency of spin waves, but it significantly influences the intensity of spin waves. The study of magnetic ion doping in $\mathrm{MnF}_{2}$ showed that the intensity of "localized" spin wave is roughly proportional to impurity concentration, and it is about five times smaller than the intrinsic spin wave with $1 \%-2 \%$ doping $[125,126]$. Our recent study of non-magnetic ion doping of hexagonal manganites showed that the intensity of intrinsic spin wave decreases much faster than the increase of doping concentration [128]. This suggests that when the intrinsic spin wave propagates to a non-magnetic ion, it will be quenched. This character would be helpful for controlling the propagation direction of spin wave by designing the pattern of doped non-magnetic ions. 
Although not helpful for tuning spin wave frequency, the doping studies suggested important properties of spin wave: intrinsic spin wave could only be excited in the host magnetic ion network; impurity related "localized" spin wave could only be excited in the impurity site; when intrinsic spin wave propagates to a non-magnetic ion, it will be quenched. These properties of spin wave would be helping for designing specific magnetic crystal structures for controlling spin wave. The artificial crystal structures have been attracted tremendous research efforts; the well studied being optical photonic crystals and acoustic phononic crystals [138-152]. The third category of artificial magnetic crystal structures, so-called magnonic crystals, are the magnetic analog of photonic crystals and phononic crystals and are useful for manipulating of spin wave. This newcomer has not only played a pivotal role in the emergence of the field of magnonics, but also is more and more widely appreciated as a unique and valuable tool in the study of general wave dynamics in metamaterial systems [153-160].

We propose the following two types of magnonic crystal structures by magnetic ion doping and non-magnetic ion doping for controlling spin wave. The structure of magnetic ion doping magnonic crystal is schematically presented in Figure 5a. The black dots represent host magnetic ions and the colored dots represent doped magnetic ions (different colors represent different types of magnetic ions). In this magnonic crystal, multiple frequency spin waves could be propagating simultaneously, and a specific frequency spin wave could be controlled to propagate along the doped magnetic ion path. The structure of non-magneic ion doping magnonic crystal is schematically presented in Figure $5 b$, the black dots represent host magnetic ions, the grey dots represent doped non-magnetic ions. In this magnonic crystal, the propagation direction can be controlled, i.e., in the left region of magnonic crystal, the spin wave can be controlled to propagate to left direction; in the right region of magnonic crystal, the spin wave can be controlled to propagate to right direction; and in the middle region of magnonic crystal, standing spin wave would be produced.

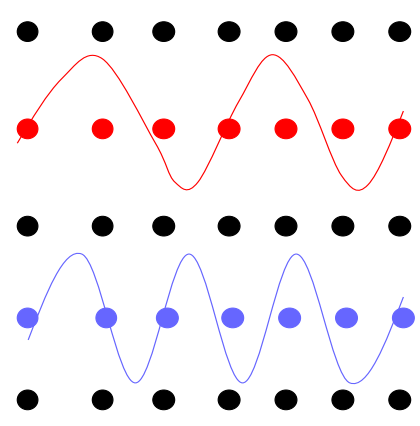

(a)

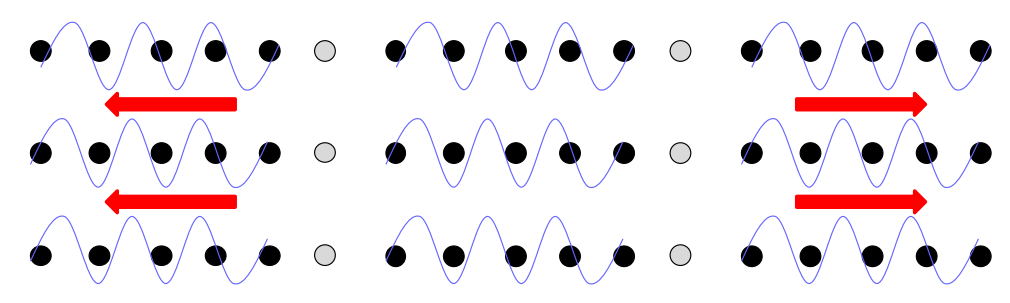

(b)

Figure 5. Proposed magnonic crystal structures by magnetic ion doping (a) and non-magnetic ion doping (b) for controlling spin wave.

\section{Summary}

In summary, the anomalous behaviors of spin waves, such as large asymmetry of anti-Stokes to Stokes intensity ratio, broad linewidth, strong resonance effect, unique polarization selection, and abnormal impurity dependence have been reviewed. These anomalous behaviors of spin waves can be 
explained by our proposed model of the processes of spin flip, spin relaxing, and spin wave scattering. Our review of the anomalous behaviors of spin waves and the proposed models provide the directions for future systematic experimental studies of spin waves by inelastic light scattering. It will be useful for raising the research interests for investigating spin waves, which is important for the emerging research field of magnonics. Also, through the analysis of abnormal impurity dependence of spin waves, two types of magnonic crystal structures by magnetic ion doping and non-magnetic ion doping have been proposed for manipulating and controlling spin waves, which would be useful in future development of magnonic devices.

Author Contributions: Investigation, H.-Y.H., M.Y., and J.Q.; resources, H.-Y.H., M.Y., and J.Q.; writing-original draft preparation, H.-Y.H.; writing-review and editing, X.-B.C. and I.-S.Y.; project administration, X.-B.C. and I.-S.Y.; funding acquisition, X.-B.C. and I.-S.Y.

Funding: This research was funded by the National Natural Science Foundation of China (Grant No. 11574241) and the National Research Foundation of Korea (NRF) grant funded by the Korean Ministry of Science and ICT (No. 2017R1A2B2009309).

Conflicts of Interest: The authors declare no conflict of interest.

\section{References}

1. Kruglyak, V.V.; Demokritov, S.O.; Grundler, D. Magnonics. J. Phys. D Appl. Phys. 2010, 43, 264001. [CrossRef]

2. Neusser, S.; Grundler, D. Magnonics: Spin waves on the nanoscale. Adv. Mater. 2009, 21, 2927. [CrossRef]

3. Rovillain, P.; De Sousa, R.; Gallais, Y.; Sacuto, A.; Méasson, M.A.; Colson, D.; Forget, A.; Bibes, M.; Barthélémy, A.; Cazayous, M. Electric-field control of spin waves at room temperature in multiferroic $\mathrm{BiFeO}_{3}$. Nat. Mater. 2010, 9, 975-979. [CrossRef] [PubMed]

4. Lenk, B.; Ulrichs, H.; Garbs, F.; Münzenberg, M. The building blocks of magnonics. Phys. Rep. 2011, 507, 107-136. [CrossRef]

5. Demokritov, S.O.; Slavin, A.N. Magnonics, from Fundamental to Applications; Springer: Berlin/Heidelberg, Germany, 2013.

6. Stamps, R.L.; Breitkreutz, S.; Åkerman, J.; Chumak, A.V.; Otani, Y.C.; Bauer, G.E.W.; Thiele, J.U.; Bowen, M.; Majetich, S.A.; Mathias Kläui, M.; et al. The 2014 magnetism roadmap. J. Phys. D Appl. Phys. 2014, 47, 333001. [CrossRef]

7. Chumak, A.V.; Serga, A.A.; Hillebrands, B. Magnon transistor for all-magnon data processing. Nat. Commun. 2014, 5, 4700. [CrossRef]

8. Chumak, A.V.; Vasyuchka, V.I.; Serga, A.A.; Hillebrands, B. Magnon spintronics. Nat. Phys. 2015, 11, 453. [CrossRef]

9. Chen, W.; Sigrist, M. Dissipationless multiferroic magnonics. Phys. Rev. Lett. 2015, 114, 157203. [CrossRef]

10. Oh, J.; Le, M.D.; Nahm, H.H.; Sim, H.; Jeong, J.; Perring, T.G.; Woo, H.; Nakajima, K.; Ohira-Kawamura, S.; Yamani, Z.; et al. Spontaneous decays of magneto-elastic excitations in non-collinear antiferromagnet (Y, $\mathrm{Lu}) \mathrm{MnO}_{3}$. Nat. Commun. 2016, 7, 13146. [CrossRef]

11. Grundler, D. Nanomagnonics around the corner. Nat. Nanotech. 2016, 11, 407. [CrossRef]

12. Chumak, A.V.; Schultheiss, H. Magnonics: Spin waves connecting charges, spins and photons. J. Phys. D Appl. Phys. 2017, 50, 300201. [CrossRef]

13. Garst, M.; Waizner, J.; Grundler, D. Collective spin excitations of helices and magnetic skyrmions: Review and perspectives of magnonics in non-centrosymmetric magnets. J. Phys. D Appl. Phys. 2017, 50, 293002. [CrossRef]

14. Yu, H.; Xiao, J.; Pirro, P. Magnonspintronics. J. Magn. Magn. Mater. 2018, 450, 1-2. [CrossRef]

15. Wang, X.S.; Zhang, H.W.; Wang, X.R. Topological Magnonics: A Paradigm for Spin-Wave Manipulation and Device Design. Phys. Rev. Appl. 2018, 9, 024029. [CrossRef]

16. Sadovnikov, A.V.; Odintsov, S.A.; Beginin, E.N.; Sheshukova, S.E.; Sharaevskii, Y.P.; Nikitov, S.A. Toward nonlinear magnonics: Intensity-dependent spin-wave switching in insulating side-coupled magnetic stripes. Phys. Rev. B 2017, 96, 144428. [CrossRef] 
17. Sadovnikov, A.V.; Grachev, A.A.; Sheshukova, S.E.; Sharaevskii, Y.P.; Serdobintsev, A.A.; Mitin, D.M.; Nikitov, S.A. Magnon straintronics: Reconfigurable spin-wave routing in strain-controlled bilateral magnetic stripes. Phys. Rev. Lett. 2018, 120, 257203. [CrossRef] [PubMed]

18. Sadovnikov, A.V.; Grachev, A.A.; Beginin, E.N.; Sheshukova, S.E.; Sharaevskii, Y.P.; Nikitov, S.A. Voltage-controlled spin-wave coupling in adjacent ferromagnetic-ferroelectric heterostructures. Phys. Rev. Appl. 2017, 7, 014013. [CrossRef]

19. Sadovnikov, A.V.; Gubanov, V.A.; Sheshukova, S.E.; Sharaevskii, Y.P.; Nikitov, S.A. Spin-wave drop filter based on asymmetric side-coupled magnonic crystals. Phys. Rev. Appl. 2018, 9, 051002. [CrossRef]

20. Sadovnikov, A.V.; Grachev, A.A.; Gubanov, V.A.; Odintsov, S.A.; Martyshkin, A.A.; Sheshukova, S.E.; Sharaevskii, Y.P.; Nikitov, S.A. Spin-wave intermodal coupling in the interconnection of magnonic units. Appl. Phys. Lett. 2018, 112, 142402. [CrossRef]

21. Vaz, C.A.F. Electric field control of magnetism in multiferroic heterostructures. J. Phys. Condens. Matter. 2012, 24, 333201. [CrossRef]

22. De Sousa, R.; Moore, J.E. Electrical control of magnon propagation in multiferroic $\mathrm{BiFeO}_{3}$ films. Appl. Phys. Lett. 2008, 92, 022514. [CrossRef]

23. Matsukura, F.; Tokura, Y.; Ohno, H. Control of magnetism by electric fields. Nat. Nanotechnol. 2015, 10, 209-220. [CrossRef] [PubMed]

24. De Sousa, R.; Allen, M.; Cazayous, M. Theory of Spin-Orbit Enhanced Electric-Field Control of Magnetism in Multiferroic $\mathrm{BiFeO}_{3}$. Phys. Rev. Lett. 2013, 110, 267202. [CrossRef] [PubMed]

25. Cherepov, S.; Amiri, P.K.; Alzate, J.G.; Wong, K.; Lewis, M.; Upadhyaya, P.; Nath, J.; Bao, M.; Bur, A.; Wu, T.; et al. Electric-field-induced spin wave generation using multiferroicmagnetoelectric cells. Appl. Phys. Lett. 2014, 104, 082403. [CrossRef]

26. Sadovnikov, A.V.; Beginin, E.N.; Bublikov, K.V.; Grishin, S.V.; Sheshukova, S.E.; Sharaevskii, Y.P.; Nikitov, S.A. Brillouin light scattering study of transverse mode coupling in confined yttrium iron garnet/barium strontium titanate multiferroic. J. Appl. Phys. 2015, 118, 203906. [CrossRef]

27. Sadovnikov, A.V.; Beginin, E.N.; Sheshukova, S.E.; Sharaevskii, Y.P.; Stognij, A.I.; Novitski, N.N.; Sakharov, V.K.; Khivintsev, Y.V.; Nikitov, S.A. Route toward semiconductor magnonics: Light-induced spin-wave nonreciprocity in a YIG/GaAs structure. Phys. Rev. B 2019, 99, 054424. [CrossRef]

28. Sadovnikov, A.V.; Bublikov, K.V.; Beginin, E.N.; Nikitov, S.A. The electrodynamic characteristics of a finite-width metal/dielectric/ferroelectric/dielectric/metal layer structure. J. Commun. Technol. Electron. 2014, 59, 914-919. [CrossRef]

29. Kuzmenko, A.M.; Szaller, D.; Kain, T.; Dziom, V.; Weymann, L.; Shuvaev, A.; Pimenov, A.; Mukhin, A.A.; Ivanov, V.U.; Gudim, I.A.; et al. Switching of magnons by electric and magnetic fields in multiferroic borates. Phys. Rev. Lett. 2018, 120, 027203. [CrossRef]

30. Rado, G.T.; Weertman, J.R. Spin-wave resonance in a ferromagnetic metal. J. Phys. Chem. Solids 1959, 11, 315-333. [CrossRef]

31. Flovik, V.; Pettersen, B.H.; Wahlström, E. Eddy-current effects on ferromagnetic resonance: Spin wave excitations and microwave screening effects. J. Appl. Phys. 2016, 119, 163903. [CrossRef]

32. Vazhenina, I.G.; Iskhakov, R.S.; Chekanova, L.A. Spin-wave resonance in chemically deposited Fe-Ni films: Measuring the spin-wave stiffness and surface anisotropy constant. Phys. Solid State 2018, 60, $292-298$. [CrossRef]

33. Liu, X.; Li, X.; Bac, S.K.; Zhang, X.; Dong, S.; Lee, S.; Dobrowolska, M.; Furdyna, J.K. Ferromagnetic resonance and spin-wave resonances in GaMnAsP films. AIP Adv. 2018, 8, 056402. [CrossRef]

34. Gopman, D.B.; Lau, J.W.; Mohanchandra, K.P.; Wetzlar, K.; Carman, G.P. Determination of the exchange constant of $\mathrm{Tb}_{0.3} \mathrm{Dy}_{0.7} \mathrm{Fe}_{2}$ by broadband ferromagnetic resonance spectroscopy. Phys. Rev. B 2016, 93, 064425. [CrossRef] [PubMed]

35. Gokhale, M.P.; Mills, D.L. Spin excitations of a model itinerant ferromagnetic film: Spin waves, Stoner excitations, and spin-polarized electron-energy-loss spectroscopy. Phys. Rev. B 1994, 49, 3880-3893. [CrossRef] [PubMed]

36. Plihal, M.; Mills, D.L.; Kirschner, J. Spin wave signature in the spin polarized electron energy loss spectrum of ultrathin Fe films: Theory and experiment. Phys. Rev. Lett. 1999, 82, 2579-2582. [CrossRef] 
37. Vollmer, R.; Etzkorn, M.; Kumar, P.S.A.; Ibach, H.; Kirschner, J. Spin-polarized electron energy loss spectroscopy of high energy, large wave vector spin waves in ultrathin FCC Co films on Cu (001). Phys. Rev. Lett. 2003, 91, 147201. [CrossRef] [PubMed]

38. Prokop, J.; Tang, W.X.; Zhang, Y.; Tudosa, I.; Peixoto, T.R.F.; Zakeri, K.; Kirschner, J. Magnons in a ferromagnetic monolayer. Phys. Rev. Lett. 2009, 102, 177206. [CrossRef] [PubMed]

39. Zakeri, K.; Zhang, Y.; Kirschner, J. Surface magnons probed by spin-polarized electron energy loss spectroscopy. J. Electron Spectrosc. Relat. Phenom. 2013, 189, 157-163. [CrossRef]

40. Hutchings, M.T.; Samuelsen, E.J. Measurement of spin-wave dispersion in NiO by inelastic neutron scattering and its relation to magnetic properties. Phys. Rev. B 1972, 6, 3447-3461. [CrossRef]

41. Bohn, H.G.; Zinn, W.; Dorner, B.; Kollmar, A. Neutron scattering study of spin waves and exchange interactions in ferromagnetic EuS. Phys. Rev. B 1980, 22, 5447. [CrossRef]

42. Mook, H.A.; Paul, D.M.K. Neutron-scattering measurement of the spin-wave spectra for nickel. Phys. Rev. Lett. 1985, 54, 227-229. [CrossRef] [PubMed]

43. Rossat-Mignod, J.; Regnault, L.P.; Vettier, C.; Bourges, P.; Burlet, P.; Bossy, J.; Henry, J.Y.; Lapertot, G. Neutron scattering study of the $\mathrm{YBa}_{2} \mathrm{Cu}_{3} \mathrm{O}_{6+x}$ system. Physica C 1991, 185-189, 86-92. [CrossRef]

44. Huberman, T.; Coldea, R.; Cowley, R.A.; Tennant, D.A.; Leheny, R.L.; Christianson, R.J.; Frost, C.D. Two-magnon excitations observed by neutron scattering in the two-dimensional spin-5/2 Heisenberg antiferromagnet $\mathrm{Rb}_{2} \mathrm{MnF}_{4}$. Phys. Rev. B 2005, 72, 014413. [CrossRef]

45. Ran, K.; Wang, J.; Wang, W.; Dong, Z.; Ren, X.; Bao, S.; Li, S.; Ma, Z.; Gan, Y.; Zhang, Y.; et al. Spin-Wave Excitations Evidencing the Kitaev Interaction in Single Crystalline a-RuCl 3 . Phys. Rev. Lett. 2017, 118, 107203. [CrossRef] [PubMed]

46. Golosovsky, I.V.; Ovsyanikov, A.K.; Aristov, D.N.; Matveeva, P.G.; Mukhin, A.A.; Boehm, M.; Regnault, L.P.; Bezmaternykh, L.N. Spin-wave dynamics and exchange interactions in multiferroicNdFe $3(\mathrm{BO} 3)_{4}$ explored by inelastic neutron scattering. J. Magn. Magn. Mater. 2018, 451, 443-449. [CrossRef]

47. Fleury, P.A.; Porto, S.P.S.; Cheesman, L.E.; Guggenheim, H.J. Light Scattering by Spin Waves in FeF 2 . Phys. Rev. Lett. 1966, 17, 84-87. [CrossRef]

48. Fleury, P.A.; Porto, S.P.S.; Loudon, R. Two-Magnon Light Scattering in Antiferromagnetic. Phys. Rev. Lett. 1967, 18, 658-662. [CrossRef]

49. Fleury, P.A. Evidence for Magnon-Magnon Interactions in $\mathrm{RbMnF}_{3}$. Phys. Rev. Lett. 1968, 21, 151. [CrossRef]

50. Fleury, P.A.; Guggenheim, H.J. Magnon-Pair Modes in Two Dimensions. Phys. Rev. Lett. 1970, $25,197$. [CrossRef]

51. Cottam, M.G. On the temperature dependence of one-magnon light scattering in antiferromagnets. J. Phys. C Solid State Phys. 1975, 8, 1933-1949. [CrossRef]

52. Bates, P.A.; Cottam, M.G.; Smith, S.R.P. “Anomalous” behaviour of the anti-Stokes-Stokes intensity ratio for one-magnon Raman scattering in $\mathrm{FeF}_{2}$. Solid State Commun. 1980, 33, 129-132. [CrossRef]

53. Petrou, A.; Peterson, D.L.; Venugopalan, S.; Galazka, R.R.; Ramdas, A.K.; Rodriguez, S. Zeeman Effect of the Magnetic Excitations in a Diluted Magnetic Semiconductor: A Raman Scattering Study of $\mathrm{Cd}_{1-\mathrm{x}} \mathrm{Mn}_{\mathrm{x}} \mathrm{Te}$. Phys. Rev. Lett. 1982, 48, 1036-1039. [CrossRef]

54. Rezende, S.M.; De Aguiar, F.M.; Lucena, M.A.; Azevedo, A. Magnon Excitation by Spin Injection in Thin Fe/Cr/Fe Films. Phys. Rev. Lett. 2000, 84, 4212-4215. [CrossRef] [PubMed]

55. Cazzanelli, E.; Kuzmin, A.; Mironova-Ulmane, N.; Mariotto, G. Behavior of one-magnon frequency in antiferromagnetic $\mathrm{Ni}_{\mathrm{c}} \mathrm{Mg}_{1-\mathrm{c}} \mathrm{O}$ solid solutions. Phys. Rev. B 2005, 71, 134415. [CrossRef]

56. Meloche, E.; Cottam, M.G.; Gnezdilov, V.P.; Lockwood, D.J. One-magnon inelastic light scattering in the canted antiferromagnetNiF 2 . Phys. Rev. B 2010, 81, 024426. [CrossRef]

57. Di, K.; Zhang, V.L.; Lim, H.S.; Ng, S.C.; Kuok, M.H.; Yu, J.; Yoon, J.; Qiu, X.; Yang, H. Direct observation of the Dzyaloshinskii-Moriya interaction in a Pt/Co/Ni film. Phys. Rev. Lett. 2015, 114, 047201. [CrossRef] [PubMed]

58. Nguyen, T.M.H.; Sandilands, L.J.; Sohn, C.H.; Kim, C.H.; Wysocki, A.L.; Yang, I.S.; Moon, S.J.; Ko, J.H.; Yamaura, J.; Hiroi, Z.; et al. Two-magnon scattering in the $5 \mathrm{~d}$ all-in-all-out pyrochlore magnet $\mathrm{Cd}_{2} \mathrm{Os}_{2} \mathrm{O}_{7}$. Nat. Commun. 2015, 8, 251. [CrossRef]

59. Gretarsson, H.; Sung, N.H.; Höppner, M.; Kim, B.J.; Keimer, B.; Tacon, M.L. Two-Magnon Raman Scattering and Pseudospin-Lattice Interactions in $\mathrm{Sr}_{2} \mathrm{IrO}_{4}$ and $\mathrm{Sr}_{3} \mathrm{Ir}_{2} \mathrm{O}_{7}$. Phys. Rev. Lett. 2016, 116, 136401. [CrossRef] 
60. Odintsov, S.A.; Sadovnikov, A.V.; Grachev, A.A.; Beginin, E.N.; Sharaevskii, Y.P.; Nikitov, S.A. Spatial-frequency selection of magnetostatic waves in a two-dimensional magnonic crystal lattice. JETP Lett. 2016, 104, 563-567. [CrossRef]

61. Wettling, W.; Cottam, M.G.; Sandercock, J.R. The relation between one-magnon light scattering and the complex magneto-optic effects in YIG. J. Phys. C Solid State Phys. 1975, 8, 211. [CrossRef]

62. White, R.M.; Nemanich, R.J.; Herring, C. Light scattering from magnetic excitations in orthoferrites. Phys. Rev. B 1982, 25, 1822-1836. [CrossRef]

63. Camley, R.E.; Grünberg, P.; Mayr, C.M. Stokes-Anti-Stokes asymmetry in Brillouin scattering from magnons in thin ferromagnetic films. Phys. Rev. B 1982, 26, 2609-2614. [CrossRef]

64. Petrou, A.; Peterson, D.L.; Venugopalan, S.; Galazka, R.R.; Ramdas, A.K.; Rodriguez, S. Raman scattering study of the magnetic excitations in diluted magnetic semiconductors in the presence of an external magnetic field. Phys. Rev. B 1983, 27, 3471-3482. [CrossRef]

65. Lockwood, D.J.; Cottam, M.G.; So, V.C.Y.; Katiyar, R.S. Raman scattering from one-magnon excitations in $\mathrm{FeF}_{2}$. J. Phys. C Solid State Phys. 1984, 17, 6009-6026. [CrossRef]

66. Zivieri, R.; Vavassori, P.; Giovannini, L.; Nizzoli, F.; Fullerton, E.E.; Grimsditch, M.; Metlushko, V. Stokes-Anti-Stokes Brillouin intensity asymmetry of spin-wave modes in ferromagnetic films and multilayers. Phys. Rev. B 2002, 65, 165406. [CrossRef]

67. Crew, D.C.; Stamps, R.L.; Liu, H.Y.; Wang, Z.K.; Kuok, M.H.; Ng, S.C.; Barmak, K.; Kim, J.; Lewis, L.H. Light scattering from spin wave excitations in a Co/CoPt exchange spring. J. Magn. Magn. Mater. 2005, 290, 530-532. [CrossRef]

68. Stashkevich, A.A.; Roussigné, Y.; Djemia, P.; Chérif, S.M.; Evans, P.R.; Murphy, A.P.; Hendren, W.R.; Atkinson, R.; Pollard, R.J.; Zayats, A.V.; et al. Spin-wave modes in Ni nanorod arrays studied by Brillouin light scattering. Phys. Rev. B 2009, 80, 144406. [CrossRef]

69. Jantzt, W.; Sandercock, J.R.; Wettling, W. Determination of magnetic and elastic properties of $\mathrm{FeBO}_{3}$ by light scattering. J. Phys. C Solid State Phys. 1976, 9, 2229-2240. [CrossRef]

70. Venugopalan, S.; Becker, M.M. Raman scattering study of $\mathrm{LuFeO}_{3}$. J. Chem. Phys. 1990, 93, $3833-3836$. [CrossRef]

71. Grünberg, P.; Metawe, F. Light scattering from bulk and surface spin waves in EuO. Phys. Rev. Lett. 1977, 39, 1561-1565. [CrossRef]

72. Sandercock, J.; Wettling, W. Light scattering from thermal magnons in iron and nickel. IEEE Trans. Magn. 1978, 14, 442-444. [CrossRef]

73. Sandercock, J.R.; Wettling, W. Light scattering from surface and bulk thermal magnons in iron and nickel. J. Appl. Phys. 1979, 50, 7784. [CrossRef]

74. Grimsditch, M.; Malozemoff, A.; Brunsch, A. Standing Spin Waves Observed by Brillouin Scattering in Amorphous Metallic Films. Phys. Rev. Lett. 1979, 43, 711. [CrossRef]

75. Malozemoff, A.P.; Grimsditch, M.; Aboaf, J.; Brunsch, A. Brillouin-scattering studies of polycrystalline and amorphous sputtered films of $\mathrm{Fe}_{1-x} \mathrm{~B}_{x}$ and $\mathrm{Co}_{1-x} \mathrm{~B}_{x}$. J. Appl. Phys. 1979, 50, 5885. [CrossRef]

76. Basistyy, R.; Stanislavchuk, T.N.; Sirenko, A.A.; Litvinchuk, A.P.; Kotelyanskii, M.; Carr, G.L.; Lee, N.; Wang, X.; Cheong, S.W. Infrared-active optical phonons and magnetic excitations in the hexagonal manganitesRMnO $\mathrm{O}_{3}$ ( $\mathrm{R}=\mathrm{Ho}, \mathrm{Er}, \mathrm{Tm}, \mathrm{Yb}$, and Lu). Phys. Rev. B 2014, 90, 024307. [CrossRef]

77. Stanislavchuk, T.N.; Wang, Y.; Janssen, Y.; Carr, G.L.; Cheong, S.W.; Sirenko, A.A. Magnon and electromagnon excitations in multiferroic $\mathrm{DyFeO}_{3}$. Phys. Rev. B 2016, 93, 094403. [CrossRef]

78. Nakajima, T.; Tokunaga, Y.; Matsuda, M.; Dissanayake, S.; Fernandez-Baca, J.; Kakurai, K.; Taguchi, Y.; Tokura, Y.; Arima, T. Magnetic structures and excitations in a multiferroic Y-type hexaferrite $\mathrm{BaSrCo}_{2} \mathrm{Fe}_{11} \mathrm{AlO}_{22}$. Phys. Rev. B 2016, 94, 195154. [CrossRef]

79. Soda, M.; Chang, L.J.; Matsumoto, M.; Garlea, V.O.; Roessli, B.; White, J.S.; Kawano-Furukawa, H.; Masuda, T. Polarization analysis of magnetic excitation in multiferroicBa ${ }_{2} \mathrm{CoGe}_{2} \mathrm{O}_{7}$. Phys. Rev. B 2018, 97, 214437. [CrossRef]

80. Leiner, J.C.; Kim, T.; Park, K.; Oh, J.; Perring, T.G.; Walker, H.C.; Xu, X.; Wang, Y.; Cheong, S.W.; Park, J.G. Magnetic excitations in the bulk multiferroic two-dimensional triangular lattice antiferromagnet $\left(\mathrm{Lu}_{,} \mathrm{Sc}\right) \mathrm{FeO}_{3}$. Phys. Rev. B 2018, 98, 134412. [CrossRef]

81. Hien, N.T.M.; Chen, X.B.; Hoang, L.H.; Lee, D.; Jang, S.Y.; Noh, T.W.; Yang, I.S. Raman scattering studies of the magnetic ordering in hexagonal $\mathrm{HoMnO}_{3}$ thin films. J. Raman Spectrosc. 2010, 41, 983-988. [CrossRef] 
82. Chen, X.B.; Hien, N.T.M.; Lee, D.; Jang, S.Y.; Noh, T.W.; Yang, I.S. Resonant A 1 phonon and four-magnon Raman scattering in hexagonal $\mathrm{HoMnO}_{3}$ thin film. New J. Phys. 2010, 12, 073046. [CrossRef]

83. Hien, N.T.M.; Oh, S.Y.; Chen, X.B.; Lee, D.; Jang, S.Y.; Noh, T.W.; Yang, I.S. Raman scattering studies of hexagonal rare-earth $\mathrm{RMnO}_{3}(R=\mathrm{Tb}$, Dy, Ho, Er) thin films. J. Raman Spectrosc. 2011, 42, 1774-1779. [CrossRef]

84. Chen, X.B.; Hien, N.T.M.; Lee, D.; Jang, S.Y.; Noh, T.W.; Yang, I.S. Spin exchange interactions in hexagonal manganites $\mathrm{RMnO}_{3}(\mathrm{R}=\mathrm{Tb}, \mathrm{Dy}, \mathrm{Ho}, \mathrm{Er})$ epitaxial thin films. Appl. Phys. Lett. 2011, 99, 052506. [CrossRef]

85. Chen, X.B.; Hien, N.T.M.; Yang, I.S.; Lee, D.; Noh, T.W. A Raman Study of the Origin of Oxygen Defects in Hexagonal Manganite Thin Films. Chin. Phys. Lett. 2012, 29, 126103. [CrossRef]

86. Nguyen, T.H.; Hien, N.T.M.; Chen, X.B.; Yang, I.S.; Park, Y.; Jung, Y.M. 2D correlation analysis of the magnetic excitations in Raman spectra of $\mathrm{HoMnO}_{3}$. J. Mol. Struct. 2014, 1069, 280-283. [CrossRef]

87. Chen, X.B.; Hien, N.T.M.; Han, K.; Nam, J.Y.; Huyen, N.T.; Shin, S.; Wang, X.; Cheong, S.W.; Lee, D.; Noh, T.W.; et al. Study of spin-ordering and spin-reorientation transitions in hexagonal manganites through Raman spectroscopy. Sci. Rep. 2015, 5, 13366. [CrossRef] [PubMed]

88. Hien, N.T.M.; Huyen, N.T.; Chen, X.B.; Park, Y.; Jung, Y.M.; Lee, D.; Noh, T.W.; Cheong, S.W.; Yang, I.S. Correlation between magnon and magnetic symmetries of hexagonal $R_{M_{n} O_{3}}(R=\mathrm{Er}, \mathrm{Ho}, \mathrm{Lu})$. J. Mol. Struct. 2016, 1124, 103-109.

89. Chen, X.B.; Guo, P.C.; Huyen, N.T.; Kim, S.; Yang, I.S.; Wang, X.; Cheong, S.W. Spin wave and spin flip in hexagonal $\mathrm{LuMnO}_{3}$ single crystal. Appl. Phys. Lett. 2017, 110, 122405. [CrossRef]

90. Bartolo, B.D. Optical Interactions in Solids; Wiley: Hoboken, NJ, USA, 1968.

91. Klemens, P.G. Anharmonic decay of optical phonons. Phys. Rev. 1966, 148, 845-848. [CrossRef]

92. Chen, X.B.; Huso, J.; Morrison, J.L.; Bergman, L.; Purdy, A.P. Temperature response and anharmonicity of the optical phonons in GaN nanowires. J. Appl. Phys. 2005, 98, 26106. [CrossRef]

93. Maradudin, A.A. Solid State Physics, Advances in Research and Applications; Seitz, E., Turnbull, D., Eds.; Academic Press: New York, NY, USA, 1966; Volume 18, p. 273.

94. Weber, W.H.; Ford, G.W. Simple model for the linewidth of the two-magnon Raman feature observed in high-Tc superconductors. Phys. Rev. B 1989, 40, 6890-6895. [CrossRef] [PubMed]

95. Pressl, M.; Mayer, M.; Knoll, P.; Lo, S.; Hohenester, U.; Holzinger-Schweiger, E. Magnetic Raman scattering in undoped and doped antiferromagnets. J. Raman Spectrosc. 1996, 27, 343-349. [CrossRef]

96. Machtoub, L.H.; Suemoto, T.; Shimoyama, J.; Kishio, K. Relaxation study of elementary excitations in Bi2212 investigated by picosecond Raman spectroscopy. J. Raman Spectrosc. 2002, 33, 471-474. [CrossRef]

97. Elliott, R.J.; Thorpe, M.F. The effects of magnon-magnon interaction on the two-magnon spectra of antiferromagnets. J. Phys. C Solid State Phys. 1969, 2, 1630-1643. [CrossRef]

98. Parkinson, J.B. Optical properties of layer antiferromagnets with $\mathrm{K}_{2} \mathrm{NiF}_{4}$ structure. J. Phys. C Solid State Phys. 1969, 2, 2012-2021. [CrossRef]

99. Singh, R.R.P.; Fleury, P.A.; Lyons, K.B.; Sulewski, P.E. Quantitative Determination of Quantum Fluctuations in the Spin- $\frac{1}{2}$ Planar Antiferromagnet. Phys. Rev. Lett. 1989, 62, 2736. [CrossRef]

100. Sugai, S.; Sato, M.; Kobayashi, T.; Akimitsu, J.; Ito, T.; Takagi, H.; Uchida, S.; Hosoya, S.; Kajitani, T.; Fukuda, T. High-energy spin excitations in the insulating phases of high-superconducting cuprates. Phys. Rev. B 1990, 42, 1045. [CrossRef]

101. Lyons, K.B.; Fleury, P.A. Spin fluctuations in superconducting cuprates. J. Appl. Phys. 1988, 64, 6075-6080. [CrossRef]

102. Schmidt, A.B.; Pickel, M.; Donath, M.; Buczek, P.; Ernst, A.; Zhukov, V.P.; Echenique, P.M.; Sandratskii, L.M.; Chulkov, E.V.; Weinelt, M. Ultrafast magnon generation in an Fe film on Cu (100). Phys. Rev. Lett. 2010, 105, 197401. [CrossRef]

103. Zakeri, K.; Zhang, Y.; Chuang, T.H.; Kirschner, J. Magnon lifetimes on the Fe (110) surface: The role of spin-orbit coupling. Phys. Rev. Lett. 2012, 108, 197205. [CrossRef]

104. Yelon, W.B.; Berger, L. Magnon heat conduction and magnon scattering processes in Fe-Ni alloys. Phys. Rev. B 1972, 6, 1974-1985. [CrossRef]

105. Hsu, Y.; Berger, L. Magnon heat conduction and magnon lifetimes in the metallic ferromagnet $\mathrm{Fe}_{68} \mathrm{Co}_{32}$ at low temperatures. Phys. Rev. B 1976, 114, 4059-4063. [CrossRef] 
106. Souchkov, A.B.; Simpson, J.R.; Quijada, M.; Ishibashi, H.; Hur, N.; Ahn, J.S.; Cheong, S.W.; Millis, A.J.; Drew, H.D. Exchange Interaction Effects on the Optical Properties of $\mathrm{LuMnO}_{3}$. Phys. Rev. Lett. 2003, 91, 027203. [CrossRef] [PubMed]

107. Choi, W.S.; Moon, S.J.; Seo, S.S.A.; Lee, D.; Lee, J.H.; Murugavel, P.; Noh, T.W.; Lee, Y.S. Optical spectroscopic investigation on the coupling of electronic and magnetic structure in multiferroic hexagonal $\mathrm{RMnO}_{3}(\mathrm{R}=$ Gd, Tb, Dy, and Ho) thin films. Phys. Rev. B 2008, 78, 054440. [CrossRef]

108. Fleury, P.A.; Loudon, R. Scattering of light by one-and two-magnon excitations. Phys. Rev. 1968, 166, 514-530. [CrossRef]

109. Cardona, M. Light Scattering in Solid II; Cardona, M., Güntherodt, G., Eds.; Springer: New York, NY, USA, 1982.

110. Blumberg, G.; Abbamonte, P.; Klein, M.V.; Lee, W.C.; Ginsberg, D.M.; Miller, L.L.; Zibold, A. Resonant two-magnon Raman scattering in cuprate antiferromagnetic insulators. Phys. Rev. B 1996, 53, R11930-R11933. [CrossRef] [PubMed]

111. Chubukov, A.V.; Frenkel, D.M. Resonant two-magnon Raman scattering in antiferromagnetic insulators. Phys. Rev. Lett. 1995, 74, 3057. [CrossRef]

112. Hanamura, E.; Dan, N.T.; Tanabe, Y. Excitons and two-magnon Raman scattering of the strongly correlated systems $\mathrm{La}_{2} \mathrm{CuO}_{4}$ and $\mathrm{YBa}_{2} \mathrm{Cu}_{3} \mathrm{O}_{6}$. Phys. Rev. B 2000, 62, 7033-7044. [CrossRef]

113. Tohyama, T.; Onodera, H.; Tsutsui, K.; Maekawa, S. Resonant two-magnon Raman scattering and photoexcited states in two-dimensional Mott insulators. Phys. Rev. Lett. 2002, 89, 257405. [CrossRef]

114. Shastry, B.S.; Shraiman, B.I. Theory of Raman scattering in Mott-Hubbard systems. Phys. Rev. Lett. 1990, 65, 1068. [CrossRef]

115. Chen, X.B.; Choi, J.Y.; Kim, H.T. Raman spectroscopy studies of spin wave in $\mathrm{V}_{2} \mathrm{O}_{3}$ thin films. J. Phys. D Appl. Phys. 2016, 49, 465304. [CrossRef]

116. Long, D.A. Intensities in Raman spectra I.A bond polarizability theory. Proc. R. Soc. Lond. Ser. A 1953, 217, 203-221.

117. Swanson, L.R.; Maradudin, A.A. Pseudopotential calculation of the Raman tensor for homopolar semiconductors. Solid State Commun. 1970, 8, 859-865. [CrossRef]

118. Tubino, R.; Piseri, L. Raman intensities in covalent crystals: A bond-polarizability approach. Phys. Rev. $B$ 1975, 11, 5145-5152. [CrossRef]

119. Maradudin, A.A.; Burstein, E. Relation between photoelasticity, electrostriction, and first-order Raman effect in crystals of the diamond structure. Phys. Rev. 1967, 164, 1081-1099. [CrossRef]

120. Nakashima, S.; Katahama, H.; Nakakura, Y.; Mitsuishi, A.; Pal, B. Raman scattering intensity of the long-period polytypes of $\mathrm{CdI}_{2}$. Phys. Rev. B 1985, 31, 6531-6540. [CrossRef] [PubMed]

121. Nakashima, S.; Balkanski, M. Raman scattering intensities of layered crystals. Phys. Rev. B 1986, 34, 5801-5807. [CrossRef]

122. Lewtas, H.J.; Boothroyd, A.T.; Rotter, M.; Prabhakaran, D.; Müller, H.; Le, M.D.; Roessli, B.; Gavilano, J.; Bourges, P. Magnetic excitations in multiferroic $\mathrm{LuMnO}_{3}$ studied by inelastic neutron scattering. Phys. Rev. B 2010, 82, 184420. [CrossRef]

123. Muñoz, A.; Alonso, J.A.; Martínez-Lope, M.J.; Casáis, M.T.; Martínez, J.L.; Fernández-Díaz, M.T. Magnetic structure of hexagonal $\mathrm{RMnO}_{3}(\mathrm{R}=\mathrm{Y}, \mathrm{Sc})$ : Thermal evolution from neutron powder diffraction data. Phys. Rev. B 2000, 62, 9498-9510. [CrossRef]

124. Park, J.; Park, J.G.; Jeon, G.S.; Choi, H.Y.; Lee, C.; Jo, W.; Bewley, R.; McEwen, K.A.; Perring, T.G. Magnetic ordering and spin-liquid state of $\mathrm{YMnO}_{3}$. Phys. Rev. B 2003, 68, 104426. [CrossRef]

125. Oseroff, A.; Pershan, P.S. Raman Scattering from Localized Magnetic Excitations in $\mathrm{Ni}^{2+}$-and $\mathrm{Fe}^{2+}$-Doped $\mathrm{MnF}_{2}$. Phys. Rev. Lett. 1968, 21, 1593. [CrossRef]

126. Moch, P.; Parisot, G.; Dietz, R.E.; Guggenheim, H.J. Observation of Localized Magnons by Raman Scattering in Ni-Doped $\mathrm{MnF}_{2}$. Phys. Rev. Lett. 1968, 21, 1596-1599. [CrossRef]

127. Oseroff, A.; Pershan, P.S.; Kestigian, M. Raman Scattering from Localized Magnons. Phys. Rev. 1969, 188, 1046-1047. [CrossRef]

128. Nam, J.Y.; Kim, S.; Hien, N.T.M.; Chen, X.B.; Sim, H.; Park, J.G.; Lee, D.; Noh, T.W.; Yang, I.S. Nonmagnetic ion doping effect on spin excitations in hexagonal $\mathrm{RMnGaO}_{3}(\mathrm{R}=\mathrm{Y}, \mathrm{Ho}), \mathrm{A} 20190315-0717$. In Proceedings of the 11th International conference on Magnetic and Superconducting Materials, Seoul, Korea, 17-24 August 2019. 
129. Wolfram, T.; Callaway, F. Spin-wave impurity states in ferromagnets. Phys. Rev. 1963, 130, $2207-2217$. [CrossRef]

130. Takeno, S. Spin-Wave Impurity Levels in a Heisenberg Ferromagnet. Prog. Theor. Phys. 1963, 30, 731-741. [CrossRef]

131. Frikkee, E. Calculations on magnon impurity modes of a pair defect in a face-centred cubic ferromagnet. J. Phys. C Solid State. Phys. 1969, 2, 345-355. [CrossRef]

132. Parisot, G.; Allen, S.J.; Dietz, R.E.; Guggenheim, H.J.; Moyal, R.; Moch, P.; Dugautier, C. Impurity Magnons in $\mathrm{MnF}_{2}$ :Co. J. Appl. Phys. 1970, 41, 890-891. [CrossRef]

133. Galkina, E.G.; Ivanov, B.A. Magnon modes localized on defects in a two-dimensional array of magnetic microparticles with transverse anisotropy. JETP Lett. 2016, 104, 32-36. [CrossRef]

134. Krupnitska, O.; Richter, J.; Derzhko, O. Localized-magnon chains and interchain interactions. Acta Phys. Pol. A 2017, 132, 1234-1237. [CrossRef]

135. Evers, M.; Müller, C.A.; Nowak, U. Weak localization of magnons in chiral magnets. Phys. Rev. B 2018, 97, 184423. [CrossRef]

136. Arakawa, N.; Ohe, J. Weak localization of magnons in a disordered two-dimensional antiferromagnet. Phys. Rev. B 2018, 97, 020407. [CrossRef]

137. Lisiecki, F.; Rychły, J.; Kuświk, P.; Głowiński, H.; Kłos, J.W.; Groß, F.; Träger, N.; Bykova, I.; Weigand, M.; Zelent, M.; et al. Magnons in a Quasicrystal: Propagation, Extinction, and Localization of Spin Waves in Fibonacci Structures. Phys. Rev. Appl. 2019, 11, 054061. [CrossRef]

138. Joannopoulous, J.D.; Villeneuve, P.R.; Fan, S. Photonic crystals: Putting a new twist on light. Nature 1997, 386, 143-149. [CrossRef]

139. Goyal, A.K.; Dutta, H.S.; Pal, S. Recent advances and progress in photonic crystal-based gas sensors. J. Phys. D Appl. Phys. 2017, 50, 203001. [CrossRef]

140. Sahani, P.; Vijaya, R. Polarizing properties of a 2D photonic crystal slab for simultaneous in-plane and out-of-plane light incidence. J. Phys. D Appl. Phys. 2018, 51, 355101. [CrossRef]

141. Rashidi, A.; Hatef, A.; Namdar, A. On the enhancement of light absorption in vanadium dioxide/1D photonic crystal composite nanostructures. J. Phys. D Appl. Phys. 2018, 51, 375102. [CrossRef]

142. Biggs, D.R.; Marcovati, A.; Cappelli, M.A. Millimeter wave control using a plasma filled photonic crystal resonator. J. Phys. D Appl. Phys. 2019, 52, 055202. [CrossRef]

143. Vasseur, J.O.; Deymier, P.A.; Chenni, B.; Djafari-Rouhani, B.; Dobrzynski, L.; Prevost, D. Experimental and theoretical evidence for the existence of absolute acoustic band gaps in two-dimensional solid phononic crystals. Phys. Rev. Lett. 2001, 86, 3012-3015. [CrossRef]

144. Jin, Y.; Pennec, Y.; Pan, Y.; Djafari-Rouhani, B. Phononic crystal plate with hollow pillars connected by thin bars. J. Phys. D Appl. Phys. 2017, 50, 035301. [CrossRef]

145. Zhou, X.; Chen, J.; Li, Y.; Sun, Y.; Xing, Y. Thermal tuning on band gaps of 2D phononic crystals considering adhesive layers. J. Phys. D Appl. Phys. 2018, 51, 075105. [CrossRef]

146. Muzar, E.; Aval, G.A.; Stotz, J.A.H. Wet-etched phononic crystal waveguiding on GaAs. J. Phys. D Appl. Phys. 2018, 51, 044001. [CrossRef]

147. Tian, Y.; Tan, Z.; Han, X.; Li, W. Phononic crystal lens with an asymmetric scatterer. J. Phys. D Appl. Phys. 2019, 52, 025102. [CrossRef]

148. Wang, Y.; Dou, S.; Shang, L.; Zhang, P.; Yan, X.; Zhang, K.; Zhao, J.; Li, Y. Effects of Microsphere Size on the Mechanical Properties of Photonic Crystals. Crystals 2018, 8, 453. [CrossRef]

149. Sun, X.; He, C.; Liu, X.; Zou, Y.; Lu, M.; Hu, X.; Chen, Y. Photonic Topological States in a Two-Dimensional Gyrotropic Photonic Crystal. Crystals 2019, 9, 137. [CrossRef]

150. Lanoy, M.; Bretagne, A.; Leroy, V.; Leroy, V.; Tourin, A. A Phononic Crystal-Based High Frequency Rheometer. Crystals 2018, 8, 195. [CrossRef]

151. Oudich, M.; Djafari-Rouhani, B.; Bonello, B.; Pennec, Y.; Sarry, F. Phononic Crystal Made of Multilayered Ridges on a Substrate for Rayleigh Waves Manipulation. Crystals 2017, 7, 372. [CrossRef]

152. Puurtinen, T.A.; Maasilta, I.J. Low-Temperature Coherent Thermal Conduction in Thin Phononic Crystal Membranes. Crystals 2016, 6, 72. [CrossRef]

153. Gubbiotti, G.; Tacchi, S.; Madami, M.; Carlotti, G.; Adeyeye, A.O.; Kostylev, M. Brillouin light scattering studies of planar metallic magnonic crystals. J. Phys. D Appl. Phys. 2010, 43, 264003. [CrossRef] 
154. Kruglyak, V.V.; Keatley, P.S.; Neudert, A.; Hicken, R.J.; Childress, J.R.; Katine, J.A. Imaging collective magnonic modes in 2D arrays of magnetic nanoelements. Phys. Rev. Lett. 2010, 104, 027201. [CrossRef]

155. Krawczyk, M.; Mamica, S.; Mruczkiewicz, M.; Klos, J.W.; Tacchi, S.; Madami, M.; Gubbiotti, G.; Duerr, G.; Grundler, D. Magnonic band structures in two-dimensional bi-component magnonic crystals with in-plane magnetization. J. Phys. D. Appl. Phys. 2013, 46, 495003. [CrossRef]

156. Arikan, M.; Au, Y.; Vasile, G.; Ingvarsson, S.; Kruglyak, V.V. Broadband injection and scattering of spin waves in lossy width-modulated magnonic crystal waveguides. J. Phys. D Appl. Phys. 2013, 46, 135003. [CrossRef]

157. Rychły, J.; Kłos, J.W. Spin wave surface states in 1D planar magnonic crystals. J. Phys. D Appl. Phys. 2017, 50, 164004. [CrossRef]

158. Chumak, A.V.; Serga, A.A.; Hillebrands, B. Magnonic crystals for data processing. J. Phys. D Appl. Phys. 2017, 50, 244001. [CrossRef]

159. Choudhury, S.; Barman, S.; Otani, Y.C.; Barman, A. Efficient Modulation of Spin Waves in Two-Dimensional Octagonal Magnonic Crystal. ACS Nano 2017, 11, 8814-8821. [CrossRef] [PubMed]

160. Hussain, B.; Cottam, M.G. Spin waves in quasiperiodic magnonic crystal arrays of metallic nanostripes. J. Phys. D Appl. Phys. 2018, 51, 355003. [CrossRef]

(C) 2019 by the authors. Licensee MDPI, Basel, Switzerland. This article is an open access article distributed under the terms and conditions of the Creative Commons Attribution (CC BY) license (http://creativecommons.org/licenses/by/4.0/). 